\title{
Chylous Arthritis
}

\author{
G. C. DAS,* F.R.C.S.ED. ; S. B. SEN,† F.R.C.S.
}

[With Special Plate Facing Page 29]

Brit. med. f., 1968, 2, 27-29

Chylous collections in various preformed anatomical sacs of the human body resulting from lymphatic obstruction have been well recognized, and entities such as chyle thorax, chylous ascites, chylous hydrocele, etc., have been described (Chatterjee, 1965). The occurrence of a knee lesion in Pondicherry closely simulating septic arthritis in its clinical presentation, but with a bacteriologically sterile effusion and resulting in complete spontaneous recovery without any specific treatment, prompted us to investigate the possibility of chylous arthritis.

\section{Methods and Material}

Twenty-five cases of the above form of "septic" arthritis admitted to two orthopaedic departments in Pondicherry during 1965-7 were studied. Complete physical and radiological examinations were carried out. In addition to routine blood and urine examinations, blood lipid, serum protein, and blood culture were carried out. The "pus" aspirated from the joint was examined biochemically and bacteriologically. Estimation of lipids in the point aspirate presented initial difficulties, as with the addition of ether a gel was formed, trapping the lipid particles. This difficulty was solved by treating the fluid with a 3:1 alcohol-ether mixture which precipitated the proteins. The lipid in the supernatant fluid was then estimated (Oser, 1965). Histopathological examination of the inflamed synovia in 10 cases and lymphographic studies in five patients were carried out to discover abnormalities, if any, in the lymphatic system.

\section{Clinical Features}

\section{Observations}

The Table shows the age-and-sex incidence. There was no striking sex predilection or involvement of one particular side. Most patients had a unilateral lesion, and in one patient both

\begin{tabular}{l}
\multicolumn{2}{l}{ Incidence of 25 Patients with Chylous Arthritis. Age and Sex } \\
\begin{tabular}{rl|c|c|c|c|c|c}
\multicolumn{2}{c|}{ Age in years: } & $0-9$ & $10-19$ & $20-29$ & $30-39$ & $40-49$ & $\begin{array}{c}50 \text { and } \\
\text { Over }\end{array}$ \\
\hline Males .. &. & 0 & 2 & 4 & 4 & 3 & 3 \\
Females &.. & 0 & 1 & 2 & 3 & 1 & 2 \\
\hline Total &. & 0 & 3 & 6 & 7 & 4 & 5 \\
\hline
\end{tabular}
\end{tabular}

knee joints were involved. The patients attended hospital with painful swelling of the knee joint associated with fever. None of them gave any history of antecedent trauma and 20 did not have any obvious septic focus elsewhere in the body. Other evidence of filariasis, the commonest cause of lymphatic obstruction locallv, was seen in two patients, one of whom had moderate elephantiasis of the opposite limb, while the other gave a history of having been operated on for a chylous hydrocele two years previously.

The lesion starts with high fever accompanied by rigor and chill. After two to three days of fever the joint becomes painful

\footnotetext{
* Department of Orthopaedic Surgery.

+ Department of Surgery.

Jawaharlal Institute of Postgraduate Medical Education and Research, Pondicherry-6, India.
}

and swollen, with limitation of movem 2 ts. When admitted patients are toxic and their temperatures vary from 100 to $103^{\circ}$ F. (37.8 to $39.4^{\circ}$ C.). The affected joint is held semiflexed; it is grossly swollen, is hot, and very tender. All movements are restricted as a result of pain and muscle spasm. Inguinal lymph nodes are enlarged and tender. The aspirated joint fluid is thin, creamy yellow, and resembles pus.

After the patient has rested in hospital the fever subsides in from three to five days and the joint becomes less tense and painful, with evidence that local inflammation and muscle spasm are resolving. The swelling persists for 7 to 10 days after remission of fever, and is completely relieved by pressure bandages. The joint then becomes entirely painless and has a full range of movements.

Twelve of these patients have been followed up for a period of one to two years. They have had repeated effusion into the joint, but none of the subsequent attacks have been associated with high fever, signs of local inflammation, or gross limitation of movements. After three or four such attacks the synovia becomes permanently thickened and nodular and the joint chronically painful.

\section{Laboratory Investigations}

Peripheral blood smears showed mild to moderate leucocytosis, the total leucocyte count varying from 8,000 to 12,000 . There was no significant characteristic alteration in the differential count. Night blood smears were positive for microfilaria in only eight cases. Blood culture was negative and joint fluid sterile for pyogenic organisms in all cases.

The lipid content of the synovial fluid varied from one and a half to three times the normal blood lipid levels $(n=25$, mean $=$ $1,238 \mathrm{mg} . / 100 \mathrm{ml} . \pm$ S.D. $288 \mathrm{mg}$.) (Fig. 1). A control study of lipid content in synovial fluid by the method described was made in 30 patients attending the orthopaedic outpatient depart-

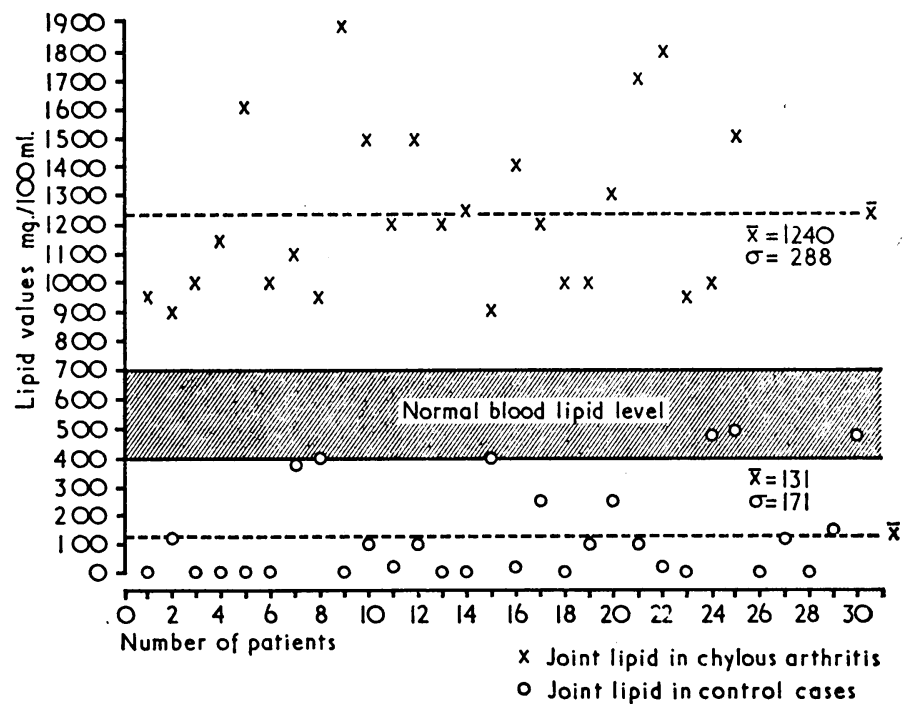

FIG. 1.-Lipid level of joint fluid in chylous arthritis and control group compared with normal blood lipid level. 
ments with effusion into the knee joint. These included cases of traumatic synovitis and gonococcal, rheumatoid, and tuberculous arthritis. The lipid content in these cases was invariably less than the normal blood level $(n=30$, mean $=131 \mathrm{mg} . /$ $100 \mathrm{ml} . \pm$ S.D. $171 \mathrm{mg}$.).

\section{Radiological Examination}

Plain $x$-ray examination of the knee joint showed a soft-tissue swelling with widening of the joint space without any abnormality or alteration in the bony contour. Lymphography was successfully carried out in five cases. In three other cases in which it was attempted the lymphatic vessels were hypoplastic and cannulation was not possible.

A lymphatic channel behind the lateral malleolus was cannulated and $5 \mathrm{ml}$. of Lipiodol Ultra-fluid injected by hand.

The afferent channel to popliteal nodes is a single normallooking vessel accompanying the short saphenous vein, which the fever and acute manifestations had subsided. In the acute phase a picture is presented resembling acute suppurative synovitis with oedema and congestion of the synovia but without any evidence of focal necrosis. High-power magnification (Special Plate, Fig. 5) shows that the polymorphs are predominant, with many lymphocytes, macrophages, few eosinophils, and plasma cells. In the later stage (Special Plate, Fig. 6) the synovium is lined by ehronic inflammatory granulation tissue with focal hyperplasia of the covering endothelium. The cellular infiltration is mostly lymphocytes with plasma cells and eosinophils.

\section{Discussion}

The occurrence of joint swelling as a result of filarial infection is well known, but the close similarity between some of these cases and septic arthritis has not been studied fully Manson-Bahr (1966) recognized acute synovitis in filariasis but offered no explanation regarding its pathogenesis. Napier (1946;

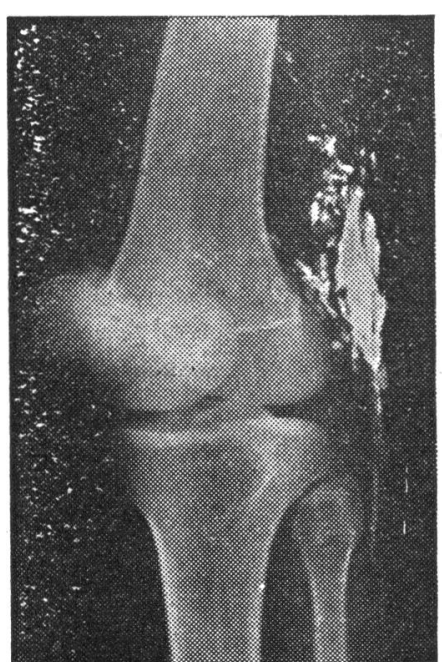

Fig. 2

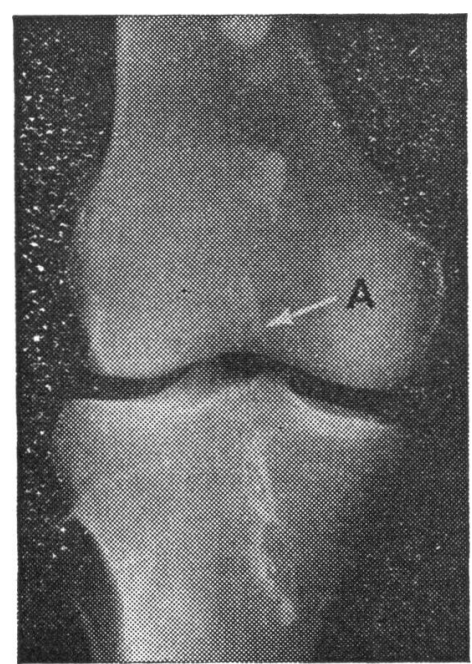

FIG. 3

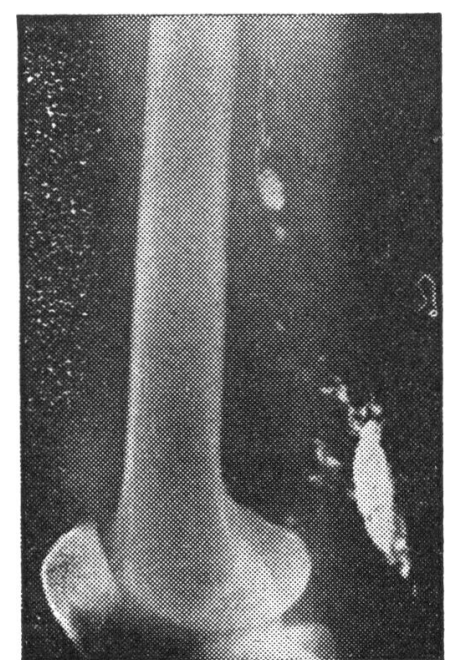

FIG. 4

Fig. 2.-Lymphogram of vessels around knee joint taken two hours after injection of dye, showing hyperplasia and varicosity of popliteal lymphatics with streaks and specks of dye in relation to the synovial reflection. FIG. 3. Lymphogram eight hours after dye injection, showing truncated blind channels leading towards the joint (A), with stasis and retrograde flow. Outline of popliteal nodes are also seen. FIG. 4.- Lymphogram 72 hours after dye injection, showing persisting residue of contrast medium in relation to the joint.

terminates in one to four popliteal nodes of normal size and structure or enlarged and spongy in appearance. The channels from these nodes always show evidence of lymphectasia, stasis, and back flow (Fig. 2). A fairly constant finding is that of short, truncated, and blind channel or channels leading towards the knee joint (Fig. 3). Abnormal collateral channels are often seen along the synovial reflexion, some of them running forwards along the margin of the suprapatellar pouch. Localized streaks and specks of contrast medium are seen in the periphery of the joint, probably in relation to the synovial membrane, particularly in the delayed films ; and stasis in these abnormally dilated lymphatic channels may be found in films taken several days later (Fig. 4).

The afferent channels in the thigh are usually tortuous and dilated, with segmental beading. These terminate in deep inguinal nodes either normal in appearance or enlarged and spongy. The pelvic and retroperitoneal nodes are fewer in number or completely absent, and are often enlarged and spongy in appearance. The afferent lymphatic channels in the pelvic system are dilated and vermiform in some cases.

\section{Histopathological Examination}

Synovial biopsy was undertaken in 10 cases. In six of these it was carried out during the acute phase when the patient was toxic and had high fever, while in four it was done after described filarial synovitis in the knee and hip as one of the complications of systemic filariasis, and suggested that it might be due to rupture of a lymph varix into the joint. Servelle et al. (1963), in one of their cases (No. 5) of extensive and proved chyle reflux with chylorrhoea and elephantiasis of a lower limb, reported a knee lesion similar to that in our cases which they in retrospect presumed to be due to chyle arthrosis. Lymphographic studies in the present series show the constant presence of demonstrable lymphectasia, stasis, and varicosity in the popliteal system of lymphatics with short blind channels leading to the joint and abnormal collaterals closely related to synovial reflexion. This constitutes the principal pathoanatomical changes in the lymphatics of the knee joint in chylous arthritis. Some of these appearances suggest the possibility of lymphatic fistulation into the synovial sac. The presence of a high concentration of lipids in the joint fluid seems to confirm the occurrence of such fistulae.

The changes in the retroperitoneal lymphatic system are identical with the pattern observed in other manifestations of chyle reflux associated with filariasis (Servelle et al., 1963 ; Kanetkar et al., 1966). Therefore we feel justified in presenting the hypothesis of "chyle reflux" as the underlying mechanism in the pathogenesis of chylous arthritis.

Lymphatic cannulation was not possible in three out of eight cases studied because of hypoplasia of lymphatic channels, and this remarkably high failure rate may indicate that association 

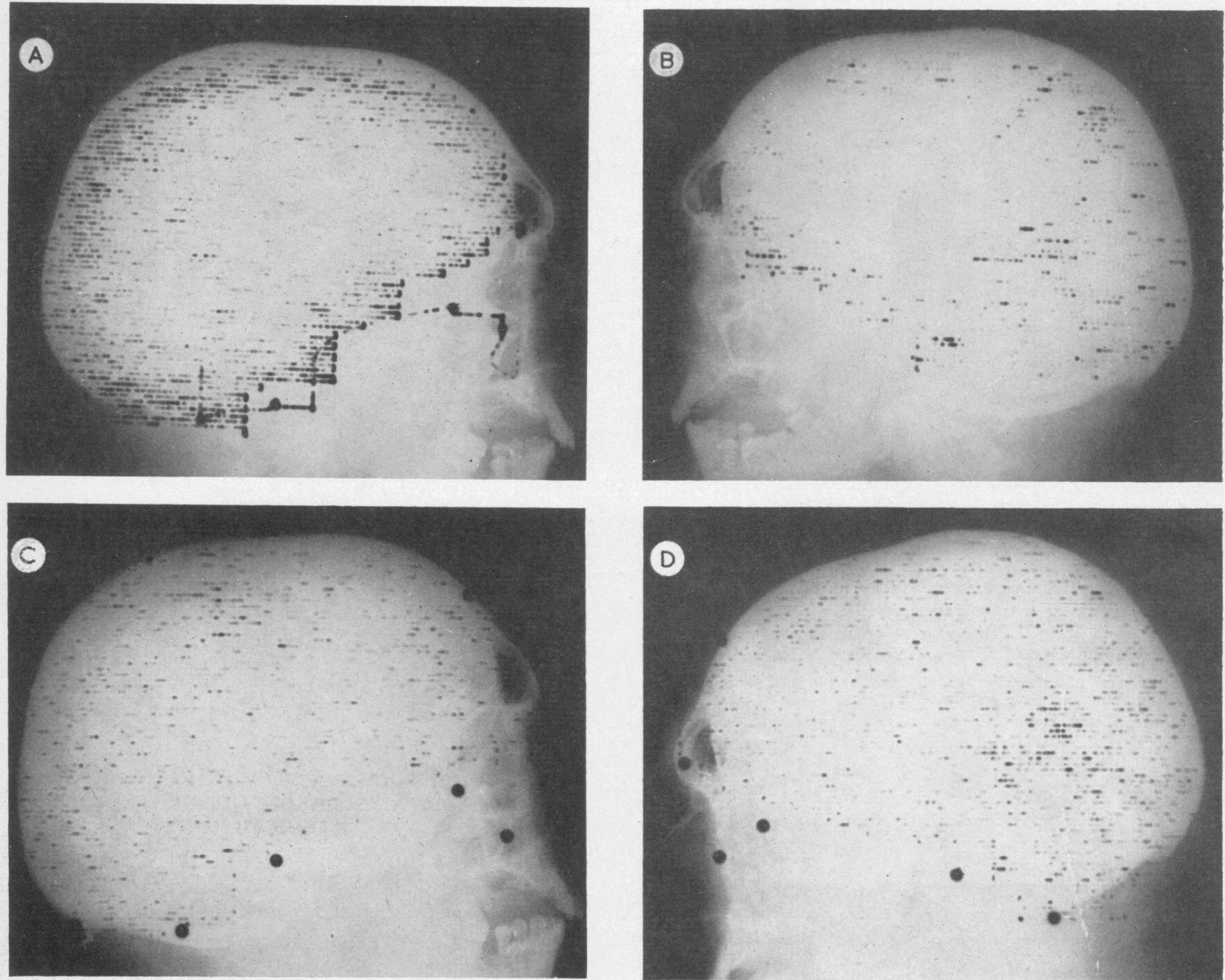

Case 7. Astrocytoma, temporo-occipital part of right hemisphere. (A) and (B): left and right lateral photoscans respectively with $113 \mathrm{~m}$ In. (C) and (D): left and right photoscans respectively with ${ }^{19}{ }^{7} \mathrm{Hg}$.

G. C. DAS AND S. B. SEN: CHYLOUS ARTHRITIS

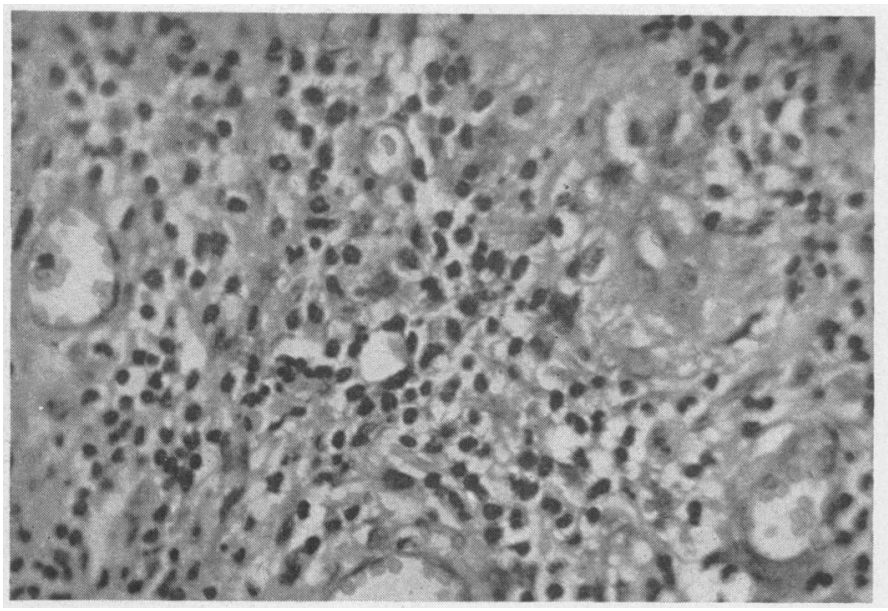

FIG. 5.-Photomicrograph of synovial lesion in the acute phase showing vascular congestion and cellular infiltration predominantly polymorphonuclear with many lymphocytes, macrophages, eosinophils, and plasma

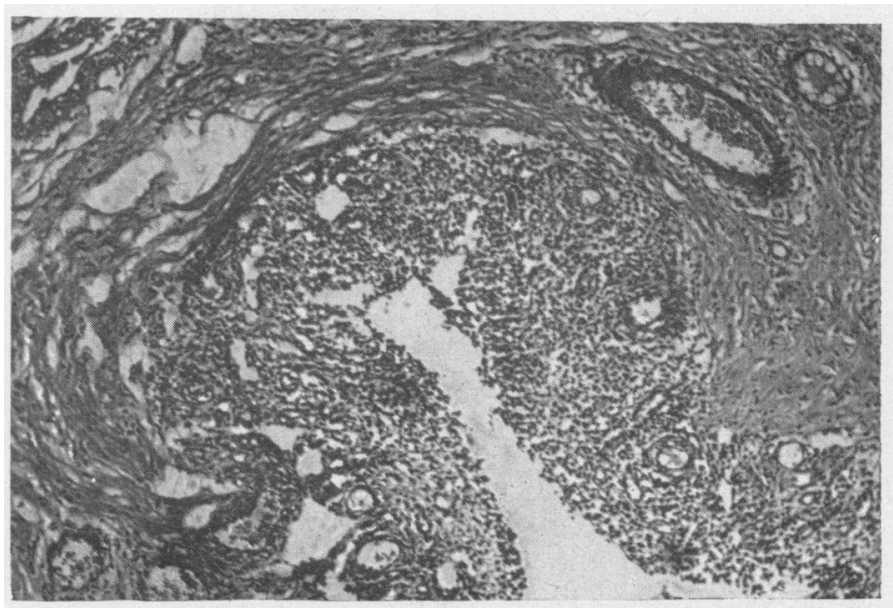

FIG. 6.-Photomicrograph of synovial lesions after remission of fever and acute symptoms, showing papillary infolding of synovium lined by chronic granulation tissue with focal hyperplasia of covering endothelium. Cellugranulation tissue with focal hyperplasia of covering endothelium. Cell
lar infiltration mostly lymphocytes with plasma cells and eosinophils. 
of hypoplasia of superficial lymphatics with chylous arthritis can have a real significance.

Several conjectures may be put forward to explain the greater frequency of the lesion in the knee joint: (1) the knee joint has the largest synovial surface and the lymphatics draining it are constantly subjected to the force of gravity, (2) greater mobility of the joint in walking, (3) lymphatics drain only to the deep system, and (4) the association of hypoplasia in the superficial system. It is hoped that further study on these lines will furnish the answer to the problem.

The presence of a large amount of chyle in the joint cavity acts as an irritant with the protein-containing chylomicron (Dole and Hamlin, 1962), setting up an allergic reaction. The presence of eosinophils and plasma cells histologically, even during the acute stage, tends to suggest such a phenomenon. The polymorphonuclear infiltration is transient and is soon taken over by lymphocytes, macrophages, eosinophils, and plasma cells in a manner similar to that of other allergic conditions. This inflammatory response leads to systemic manifestations, with fever and toxaemia, and local features of an acute inflammation with muscle spasm. In our series there is no evidence to suggest that secondary infection plays any part in its causation. The picture in this phase closely resembles that which is seen in many cases of chronic arthritis which clinically do not fit into any of the recognized groups. It is likely, therefore, that these are the result of chylous arthritis in the past.

It is important to distinguish this condition clinically from septic arthritis-often this is not easy. However, in those areas where filariasis is endemic it would be well to bear in mind the occurrence of this condition. Estimation of lipids in the joint fluid in our opinion is a safe, simple, and quick method of making a diagnosis with a sufficiently high degree of accuracy, and thereby saving a number of joints from needless arthrotomy.
In our series the prognosis of the lesions has been uniformly excellent. None of the cases developed true septic arthritis or ankylosis of the joint as reported by Manson-Bahr (1966).

\section{Summary}

The occurrence of an acute knee lesion in systemic filariasts has been studied and its clinical features are described.

The lymphographic changes described strongly suggest a phenomenon of "chyle reflux" and lymphatic fistulation and extravasation of chyle in the joint space as the mechanism underlying the pathogenesis of so-called filarial arthritis.

A method of estimating lipid in the joint fluid is described. Determination of lipid in synovial fluid is a simple and reliable method of arriving at the diagnosis.

The term "chylous arthritis" is suggested, as it describes the lesion most accurately.

We are grateful to Dr. D. J. Reddy, Principal, Jawaharlal Instjtute of Postgraduate Medical Education and Research, Pondicherry (JIPMER), and to the Medical Superintendents of the General Hospital and JIPMER Hospital, Pondicherry, for permitting us to carry out this study and the use of hospital records.

\section{REFERENCES}

Chatterjee, P. (1965). In Clinical Surgery, edited by C. Rob and R Smith, vol. 8 , edited by A. K. Basu, p. 51 . London.

Dole, V. P. and Hamlin, J. T. (1962). Physiol. Rev., 42, 674.

Kanetkar, A. V., Karanjawalla, D. K., Deshmukh, S. M., and Sen, P. K (1966). Indian f. Surg., 28, 381

Manson-Bahr, P. H. (1966). Manson's Tropical Diseases, 16th ed., p 684. London.

Napier, L. E. (1946). Principles and Practice of Tropical Medicine, $\mathbf{c}$ 674. New York

Oser, B. L. (editor) (1965). Hawk's Physiological Chemistry, 14th ed. p. 539 . New York.

Servelle, M., et al. (1963). Surgery, 54, 536.

\section{Preliminary Communications}

\section{Brainscanning with Radioindium}

[With Special Plate on facing Page]

Brit. med. F., 1968, 2, 29-30

While the advantages and reliability of utilizing radioisotopes in intracranial diagnosis are no longer disputed, doubts persist over the most suitable substance to use for this purpose. The ideal is the radioisotope with the shortest half-life that gives the maximum clinical information. Apart from ${ }^{203} \mathrm{Hg}$-chlormerodrin, which is now regarded as objectionable because of its high radiation dose, the radioisotopes most widely used for brainscanning have been ${ }^{187} \mathrm{Hg}$-chlormerodrin, ${ }^{99 \mathrm{~m}} \mathrm{Tc}$-pertechnetate, and ${ }^{131} \mathrm{I}$ human serum albumin. Stern, Goodwin, Scheffel, Wagner, and Kramer (1967) described the use in brainscanning of a suitably treated solution of ${ }^{113 m} \mathrm{In}$, which has a radioactive half-life of 1.7 hours.

In this preliminary communication a controlled clinical trial of the use of ${ }^{113 \mathrm{~m}} \mathrm{In}$ in seven patients is reported.

\section{Preparation of Material}

Preparation was similar to that described by Stern et al. (1967). ${ }^{113 m} \mathrm{In}$ was obtained by eluating a ${ }^{113} \mathrm{Sn}-{ }^{113 \mathrm{~m}} \mathrm{In}$ generator with $5.5 \mathrm{ml}$. of dilute hydrochloric acid, $\mathrm{pH} 1.35$.
To the ${ }^{113 \mathrm{~m}}$ In eluate was added $0.2 \mathrm{ml}$. of $0.1 \mathrm{~N} \mathrm{HCl}$ containing $5 \mathrm{mg}$. of $\mathrm{FeCl}_{3} \cdot 6 \mathrm{H}_{2} \mathrm{O}$ per ml., followed by $0.1 \mathrm{ml}$. of solution of D.T.P.A. (diethyl triamine penta-acetic acid chelate) containing $14 \mathrm{mg} . / \mathrm{ml}$. The mixture was then titrated to $\mathrm{pH}$ 7.5 with dilute $\mathrm{NaOH}$ and sterilized by autoclaving at $15 \mathrm{lb}$./ sq. in. $(1.05 \mathrm{~kg} . / \mathrm{sq} . \mathrm{cm}$.) for 15 minutes. Activities of up to 5 millicuries $(\mathrm{mCi})$ were given intravenously to the patient.

Stern et al. (1967) found that the urine activity two and a half hours after injection of this agent into several patients was 56-60\%. Using this figure, approximate calculations were made which showed that the radiation dose per millicurie administered is 100 millirad (mrad) to the kidney and $10 \mathrm{mrad}$ to the whole body. Corresponding radiation doses for ${ }^{197} \mathrm{Hg}$ chlormerodrin are 5 rad and 17 mrad respectively (Sodee, 1964). Thus the general radiation dose is greatly reduced by usins ${ }^{113 \mathrm{~m}}$ In-D.T.P.A.

\section{Clinical Series}

Seven patients referred for routine brainscanning with ${ }^{107} \mathrm{Hg}_{\mathrm{g}}$ were also examined with ${ }^{113 \mathrm{~m}} \mathrm{In}$. Three were rescanned with ${ }^{113 \mathrm{~m}}$ In by using different activities. Thus $10^{113 \mathrm{~m} I n}$ brainscans with three different doses were available for comparison with the seven ${ }^{197} \mathrm{Hg}$ brainscans in the seven patients. All radiological investigations in each patient were completed within seven days.

In six of the patients a firm final diagnosis was reached (Table I). In the remaining patient (Case 3), in whom a 